Check for updates

Cite this: Chem. Commun., 2020, 56,9624

Received 1st June 2020,

Accepted 17th July 2020

DOI: $10.1039 / \mathrm{d} 0 \mathrm{cc} 03834 \mathrm{~b}$

rsc.li/chemcomm

\section{Electropolymerization in a confined nanospace: synthesis of PEDOT nanoparticles in emulsion droplet reactors $\dagger$}

\author{
Myoung Won Lee, ${ }^{a}$ Deok-Jin Kwon, ${ }^{b}$ Junmo Park, ${ }^{a}$ Jae-Chul Pyun, (D c \\ Young-Joo Kim ${ }^{b}$ and Hyun S. Ahn (D) *a
}

Poly(3,4-ethylenedioxythiophene) (PEDOT) is an important material widely used in electronics for its hole conducting property. A novel strategy for the synthesis of nanoparticulate PEDOT was developed by emulsion droplet electrochemistry. Taking advantage of the space confinement in emulsions, PEDOT nanoparticles were size controllable without use of a separate template. Potential applications were investigated by implementing the PEDOT nanoparticle decorated electrodes as a supercapacitor and a hole transport layer in an organic light emitting diode.

Detection of stochastic collisions of nano-objects on an electrode has been a research topic of interest in recent years. ${ }^{1-3}$ Since the first observation of hard nanoparticle collisions by implementation of ultramicroelectrodes (UMEs), ${ }^{4}$ detection and statistical analysis of the electrode collisions of many types of nanoobjects were studied, including those of hard nanoparticles, ${ }^{5-8}$ soft nanoparticles, ${ }^{9-11}$ and biologically relevant entities. ${ }^{12-15}$ More recent adaptations of the electrochemical collision research empowered scientists to utilize these stochastic collisions as a synthetic tool. ${ }^{16-18}$ Particularly, electrochemistry of emulsions (both oil-in-water and water-in-oil) yielded metal ${ }^{19-23}$ and metal oxide $^{24}$ nanoparticles while the synthesis processes were monitored real-time by amperometry. This electrochemical synthetic strategy is particularly significant due to the precise size and composition control it provides and the surfactant-free nature of the resulting nanoparticles. ${ }^{19,20,24}$ In the present communication, we expand the capabilities of this emulsion-collision based synthetic method to polymeric nanomaterials (Scheme 1). A confined

\footnotetext{
${ }^{a}$ Department of Chemistry, Yonsei University, 50 Yonsei-ro, Seodaemun-gu, Seoul, 03722, Republic of Korea. E-mail: ahnhs@yonsei.ac.kr

${ }^{b}$ Department of Mechanical Engineering, Yonsei University, 50 Yonsei-ro, Seodaemun-gu, Seoul, 03722, Republic of Korea

${ }^{c}$ Department of Materials Science and Engineering, Yonsei University, 50 Yonsei-ro, Seodaemun-gu, Seoul, 03722, Republic of Korea

$\dagger$ Electronic supplementary information (ESI) available: Experimental details, relevant calculations, and spectral characterizations of materials. See DOI: 10.1039/d0cc03834b
}

nanospace provided by an emulsion droplet served as a synthesis template, electropolymerization in which resulted in polymeric nanoparticles of size and shape of the droplet. In the current study, oxidative electropolymerization of 3,4-ethylenedioxythiophene (EDOT) was performed to yield nanoparticulate PEDOT (polyEDOT). The synthesized particles were bound to the electrode relatively strongly for further electrochemical use, and were also detachable and dispersible in a solvent upon ultrasonic stimulation.

Polyethylenedioxythiophene is widely implemented as a hole transport layer in various electronic devices including solar cells $^{25-27}$ and light-emitting diodes..$^{28,29}$ Common preparation methods for PEDOT (including commercially available PEDOT:PSS) involve rather harsh chemical oxidants and templates. ${ }^{30-32}$ Electro-oxidative polymerization is another viable and milder synthetic method; ${ }^{33-36}$ however, electropolymerized PEDOT generally results in thin films rather than colloidal nanoparticles (NPs). Preparation of PEDOT in the form of NPs is generally more preferred due to solution processability and superior hole conductivity. ${ }^{37,38}$ A previous account reported nanoparticulate PEDOT by an electrochemical method; ${ }^{39}$ however, to the best of our knowledge, this work is the first case where discrete droplet

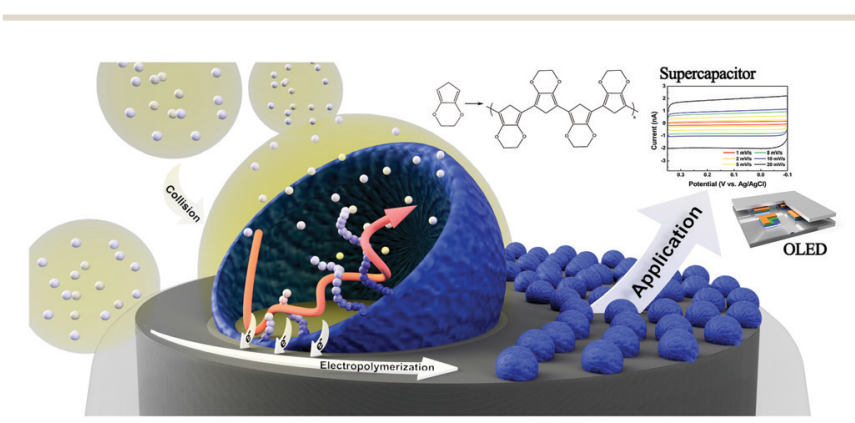

Scheme 1 Schematic representation of PEDOT electrosynthetic process by emulsion-collision method and potential applications of the PEDOT NP decorated electrodes. 
reactors were used to electrochemically synthesize PEDOT NPs with relatively narrow size distribution.

As shown in Scheme 1, the reactors employed in this work were dichloromethane emulsion droplets dispersed in aqueous continuous phase. To determine the optimum potential for the electrosynthesis experiment in emulsion droplets, EDOT electropolymerization was performed by cyclic voltammetry in dichloromethane (Fig. 1a). In the potential scale adopted in this work ( $v s . \mathrm{Ag} / \mathrm{AgCl}$ ), we observed no PEDOT formation at potentials less oxidizing than $1.15 \mathrm{~V}$, and oxidative degradation of solvent occurred at potentials more positive than $1.2 \mathrm{~V}$. Therefore, $1.15 \mathrm{~V}$ was chosen as the optimum electrode potential for the collision experiments and all of our data were collected at this potential unless otherwise stated. As commonly reported in the literature, PEDOT synthesized by cyclic voltammetry method resulted in films covering the entire electrode (Fig. 1 and Fig. S1, ESI $\dagger$ ). ${ }^{33-36,40}$

The size of the droplet reactors was controllable by varying the droplet synthesis condition (see ESI $\dagger$ ), and each droplet contained $25 \mathrm{mM}$ EDOT precursor. Oxidative electropolymerization initiated inside the droplets as they collided on the working electrode, resulting in PEDOT NPs of size concomitant to the precursor droplet, which also serves as a synthesis template (Scheme 1). The electrosynthetic process can be monitored in situ by amperometry, with each of the spike-like signals in Fig. 1 (also see Fig. S2 and S3, ESI $\dagger$ ) corresponding to electrochemical synthesis of a PEDOT NP. Due to the radical-based mechanism of the EDOT polymerization process, ${ }^{41}$ the charge under the spike-like signals does not correlate to an overall one electron oxidation of EDOT monomers contained in the droplet. The integrated charge under the spike signals (e.g. $0.42 \mathrm{pC}$ for $350 \mathrm{~nm}$ diameter droplets) was approximately ten-fold greater than that calculated for one electron oxidation per EDOT molecule $(0.054 \mathrm{pC}$ for a $350 \mathrm{~nm}$ droplet). The excess amount of charge presumably is due to oxidative degradation of the dichloromethane solvent. Considering the proximity in the potential scale of the two oxidation reactions (EDOT oxidation at $1.15 \mathrm{~V}$ and dichloromethane oxidation at $1.2 \mathrm{~V}$ ), slight oxidation of solvent was inevitable during PEDOT NP synthesis. The degree of solvent intervention was small, however, accounting for $c a$. 1\% of total solvent molecules being oxidized: dichloromethane oxidized during PEDOT (a)
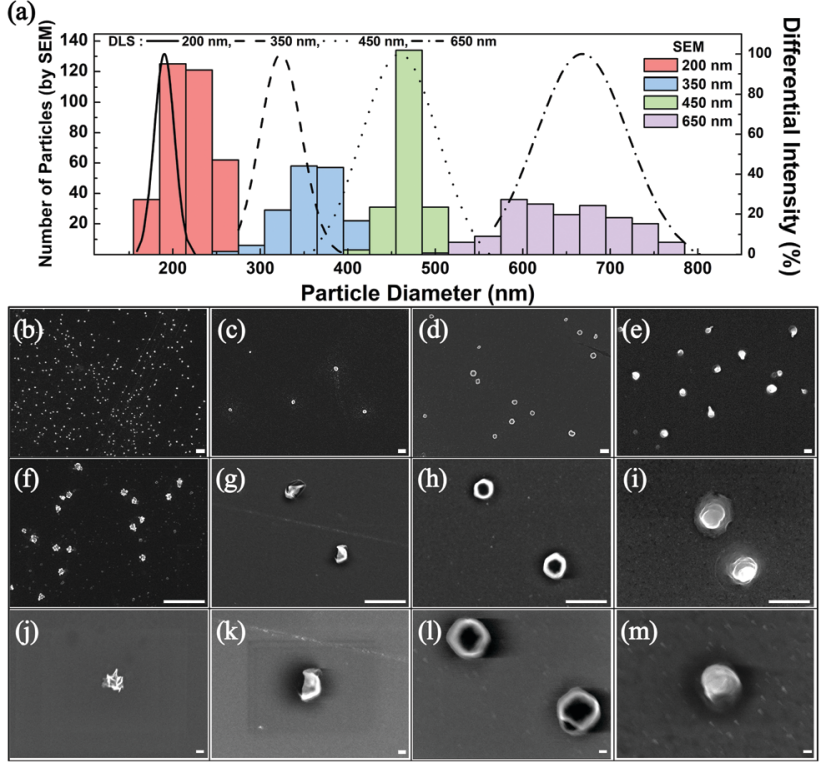

Fig. 2 (a) Histograms of PEDOT NP size measured by SEM (200 nm-red, $350 \mathrm{~nm}$-blue, $450 \mathrm{~nm}$-green, and $650 \mathrm{~nm}$-purple) and the size of the precursor droplet determined from dynamic light scattering (DLS; $200 \mathrm{~nm}$-black solid line, $350 \mathrm{~nm}$-dashed line, $450 \mathrm{~nm}$-dotted line, and $650 \mathrm{~nm}$-dash-dotted line). (b)-(m) SEM images of PEDOT NPs ((b), ( $f$ ) and (j) 200, (c), (g) and (k) 350, (d), (h) and (l) 450, and (e), (i) and (m) $600 \mathrm{~nm}$ NPs, respectively on a same magnification scale; scale bars are $1 \mu \mathrm{m}$ in length for images in (b)-(i) and $100 \mathrm{~nm}$ in images (j)-(m)).

synthesis was $c a$. $0.38 \mathrm{pC}$, whereas the amount of charge required to oxidize all solvent molecules in the droplet was $34 \mathrm{pC}$ (for a $350 \mathrm{~nm}$ diameter droplet).

The frequency of these droplet collision events can be estimated from the Stokes-Einstein equation based on the Brownian motion of the droplets in the aqueous continuous phase (see ESI $\dagger$ for detailed calculations). ${ }^{1}$ Experimentally observed frequency of events agreed well with the calculated results and are summarized in Table S1 (ESI $\dagger$ ). After several hundreds of the synthetic collision events, the electrodes were isolated and analyzed by SEM. Fig. 2 displays SEM images of the electrosynthesized PEDOT NPs obtained from droplets of various sizes: $200 \mathrm{~nm}, 350 \mathrm{~nm}, 450 \mathrm{~nm}$, and $600 \mathrm{~nm}$ in diameter. Correlation between the size of the synthesized PEDOT NPs and (a)

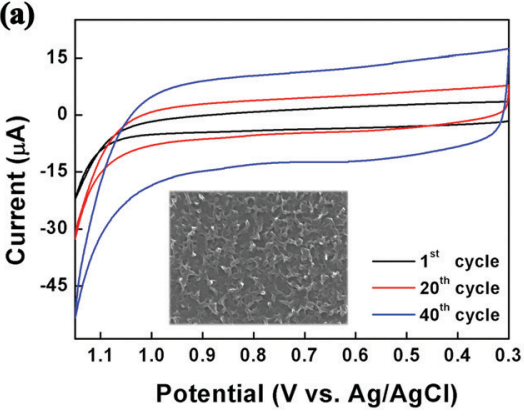

(b)

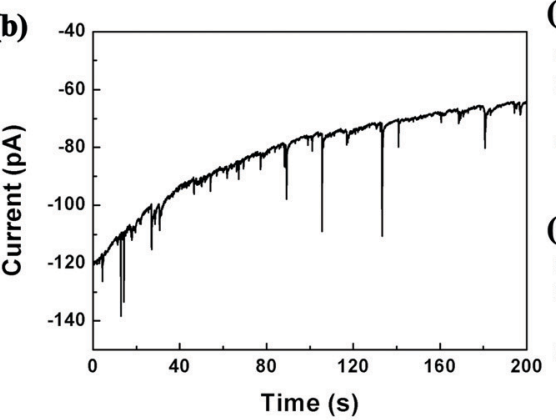

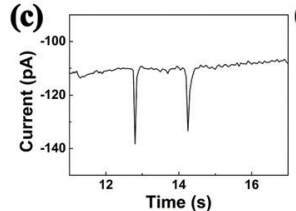

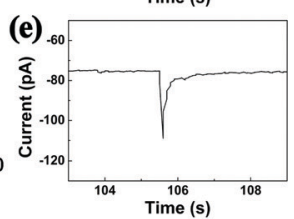

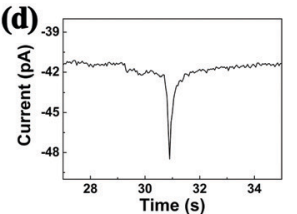

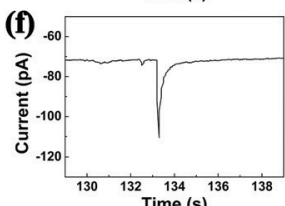

Fig. 1 (a) Electropolymerization of EDOT by cyclic voltammetry (0.1 M EDOT, $0.2 \mathrm{M}$ TBAP in DCM at $50 \mathrm{mV} \mathrm{s}^{-1}$ scan rate; inset displays resultant film by SEM). (b) Chronoamperogram exhibiting collision signals of $200 \mathrm{~nm}$ droplets onto a $10 \mu \mathrm{m}$ carbon electrode, with each oxidation spike leading to a synthesized PEDOT NP. (c)-(f) Typical single droplet electrolysis signals. See also Fig. S2 and S3 (ESI†). 
that of the precursor emulsion droplets was reasonable based on the statistical analysis of the NP size (Fig. 2), suggesting that the emulsion droplets not only serve as EDOT precursor vessels but also as NP synthesis templates. Morphology of the PEDOT NPS was in general spherical (see Fig. 2 and Fig. S4, ESI $\dagger$ ), with kinks and wrinkles typical of polymer NPs. Nanoparticles with smaller than $200 \mathrm{~nm}$ diameter were also synthesizable by this method: $c a$. 40 and $70 \mathrm{~nm}$ NPs were obtained by employing $60 \mathrm{~nm}$ and $100 \mathrm{~nm}$ droplets, respectively (see Fig. S5-S7, ESI $\dagger$ ). Particles of smaller size exhibited larger uncertainty in the measurement of their diameters in SEM due to electron beam charging at high magnifications. Adhesion of electrosynthesized PEDOT NPs onto the electrode surface was strong such that direct implementation of NP attached electrodes were feasible (vide infra; see Fig. S13 and S14, ESI $\dagger$ ). The particles were also detachable upon ultrasonic stimulation and dispersed in solvent for further processing (Fig. S8, ESI $\dagger$ ).

Oxidative polymerization process of EDOT is prevalent in the literature, and typically results exclusively in PEDOT formation without significant side reactions. ${ }^{42,43}$ In order to confirm the chemical identity of the NPs synthesized in this work, spectroscopic analyses were performed. Inspection of the NP attached electrodes by UV-vis and Raman spectroscopy revealed distinctive features corresponding to PEDOT (Fig. S9, S10 and Table S2, ESI $\dagger$ ), namely a broad absorption centered around $580 \mathrm{~nm}^{44,45}$ and vibrations corresponding to PEDOT structural motifs. ${ }^{44,45}$ Because electrosynthesized PEDOT NPs are positively charged, electrolyte anions incorporate into the NPs for charge compensation. ${ }^{4-48}$ In the case of this work, $\mathrm{PF}_{6}$ anions in the electrolyte incorporated to form PEDOT:PF 6 NPs (see also Fig. S4, ESI $\dagger$ ). Potential applications of the electrosynthesized PEDOT NPs were investigated by subjecting the NP attached electrodes as (1) a capacitor and (2) a hole transport layer in an organic light emitting diode (OLED). For capacitance measurements PEDOT NPs were synthesized on a $10 \mu \mathrm{m}$ diameter carbon UME.

As shown in Fig. 3, geometric capacitances of a carbon UME decorated with PEDOT NPs were measured. Deposition times ranging from 15 minutes to 60 minutes were chosen, corresponding to good electrode coverage of PEDOT NPs (ranging in 100 to 2500 NPs depending on size; see $\mathrm{ESI} \dagger$ for detailed calculations). Geometric capacitances of 100,160 , and $215 \mathrm{mF} \mathrm{cm}^{-1}$ were observed for $200 \mathrm{~nm}, 350 \mathrm{~nm}, 450 \mathrm{~nm}$ NP decorated electrodes, respectively. The obtained values compare favorably to literature reported capacitances of PEDOT based materials. ${ }^{49,50}$ Interestingly, UMEs decorated with larger PEDOT NPs exhibited superior capacitances compared to those with smaller NPs, which is counter intuitive considering larger surface to volume ratio of smaller NPs. We presume that larger NPs in this study (350 and $450 \mathrm{~nm}$; see Fig. S11, ESI $\dagger$ ) result in partially hollow structure, which leads to higher surface area and superior capacitor performance compared to $200 \mathrm{~nm}$ compact NPs. Capacitance values greater than $200 \mathrm{mF} \mathrm{cm} \mathrm{cm}^{-1}$ is on par or better than that of many polymer-based capacitors in the literature, ${ }^{51,52}$ and suggests that the PEDOT in this work is a promising candidate material for supercapacitor application. Supercapacitor electrodes based on
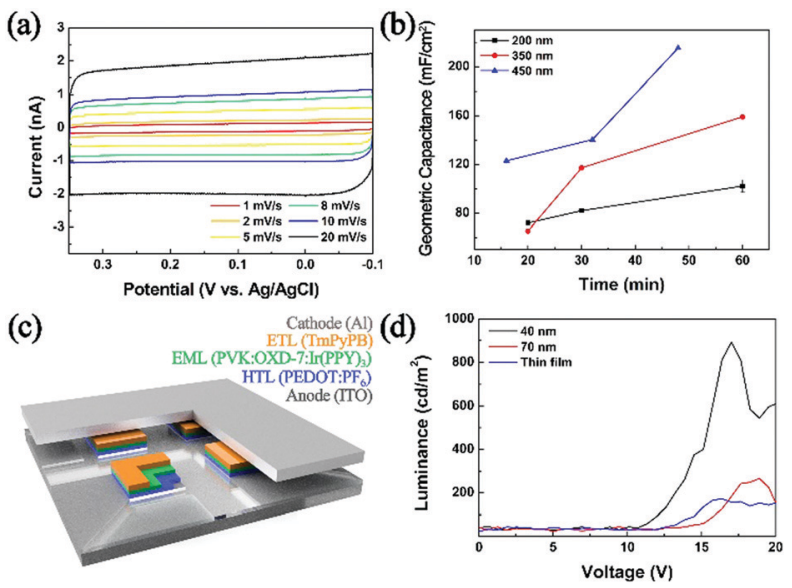

Fig. 3 (a) Cyclic voltammograms of $450 \mathrm{~nm}$ PEDOT:PF 6 (146 NPs) decorated carbon UME at different scan rates. (b) Geometric capacitance $\left(\mathrm{mF} \mathrm{cm}{ }^{-2}\right.$ ) of PEDOT NPs decorated electrodes (200 nm-black, $350 \mathrm{~nm}$-red, and $450 \mathrm{~nm}$-blue). (c) Schematic representation of an OLED device investigated in this work. (d) Luminescence measurements from OLED devices employing PEDOT:PF 6 NPs (40 and $70 \mathrm{~nm}$ ) and a PEDOT:PF 6 thin film (deposited by cyclic voltammetry) as hole transport layers.

PEDOT NPs exhibited little fade in performance even after one hundred continuous cycles and in excess of $2 \mathrm{~h}$ of continued use (Fig. S14, ESI $\dagger$ ).

Electrosynthesized NPs were also implemented as a hole transport layer in OLED devices (Fig. 3c. For detailed device preparation, see ESI; $\dagger$ configuration similar to that in ref. 53 and 54 was adopted). Devices fabricated with NPs larger than $200 \mathrm{~nm}$ did not luminesce, presumably due to circuit shortage caused by a thick hole transport layer. Typical OLED devices in the literature employ commercial PEDOT:PSS (PSS; polystyrene sulfonate) cast films of $c a .40 \mathrm{~nm}$ thickness. ${ }^{53,54}$ When we implemented PEDOT:PF 6 NPs of 40 and $70 \mathrm{~nm}$, the device luminesced, with superior performance compared to that with a thin film PEDOT:PF 6 hole transport layer (Fig. 3d). Compared to a benchmark device adopting a spin-coated commercial PEDOT:PSS hole transport layer, that with PEDOT:PF ${ }_{6}$ NPs from this work exhibited approximately 10\% luminance (Fig. S12, ESI $\dagger$ ). It is well known that PEDOT hole conductivity varies depending on the anion, and that PEDOT:PSS greatly outperforms PEDOT: $\mathrm{PF}_{6} \cdot{ }^{55-57}$ It is encouraging that the PEDOT NPS prepared in this work can actively function as a hole transport layer in a working OLED device, and that it can match $10 \%$ luminance of a device with a commercial PEDOT:PSS layer. Although in the current state, the PEDOT NP materials synthesized by the method introduced in this work are not competitive with established commercial analogue, we hope to further improve the methodology such that tuning of the counter anion may be feasible for the conductivity engineering and ultimately an optimized performance of the resulting material as a hole conducting layer in an OLED device.

In summary, we established a novel synthetic strategy towards size-controlled NPs of PEDOT by adaptation of droplet reactor collision electrochemistry. The synthesized particles adhere to the electrode strongly for direct electrochemical use 
and were also detachable and solvent dispersible upon suitable stimulation. Possible applications of PEDOT NPs were investigated in forms of supercapacitor and hole transport layer in OLED. The synthetic protocol was made possible by extension of soft nano-object collision research, and we hope to extend this strategy further towards various other polymeric nanostructures.

This work was financially supported by the National Research Foundation of Korea under grant NRF-2020R1C1C1007409.

\section{Conflicts of interest}

There are no conflicts to declare.

\section{Notes and references}

1 B.-K. Kim, A. Boika, J. Kim, J. E. Dick and A. J. Bard, J. Am. Chem. Soc., 2014, 136, 4849-4852.

2 S. E. Fosdick, M. J. Anderson, E. G. Nettleton and R. M. Crooks, J. Am. Chem. Soc., 2013, 135, 5994-5997.

3 X.-W. Zhang, A. Hatamie and A. G. Ewing, J. Am. Chem. Soc., 2020, 142, 4093-4097.

4 X. Xiao, F.-R. F. Fan, J. Zhou and A. J. Bard, J. Am. Chem. Soc., 2008, 130, 16669-16677.

5 H. Zhou, F.-R. F. Fan and A. J. Bard, J. Phys. Chem. Lett., 2010, 1, 2671-2674.

6 Y.-G. Zhou, N. V. Rees and R. G. Compton, ChemPhysChem, 2011, 12, 2085-2087.

7 X. Xiao and A. J. Bard, J. Am. Chem. Soc., 2007, 129, 9610-9612.

8 N. Daryanavard and H. R. Zare, Anal. Chem., 2017, 89, 8901-8907.

9 H. Deng, J. E. Dick, S. Kummer, U. Kragl, S. H. Strauss and A. J. Bard, Anal. Chem., 2016, 88, 7754-7761.

10 Y. Li, H. Deng, J. E. Dick and A. J. Bard, Anal. Chem., 2015, 87, 11013-11021.

11 B.-K. Kim, J. Kim and A. J. Bard, J. Am. Chem. Soc., 2015, 137, 2343-2349.

12 J. E. Dick, C. Renault and A. J. Bard, J. Am. Chem. Soc., 2015, 137, 8376-8379.

13 A. N. Sekretaryova, M. Y. Vagin, A. P. F. Turner and M. Eriksson, J. Am. Chem. Soc., 2016, 138, 2504-2507.

14 J. Y. Lee, B.-K. Kim, M. Kang and J. H. Park, Sci. Rep., 2016, 6, 30022-30027.

15 W. Cheng and R. G. Compton, Angew. Chem., Int. Ed., 2014, 53, 13928-13930.

16 D. A. Robinson and H. S. White, Nano Lett., 2019, 19, 5612-5619.

17 M. Zhou, D. Wang and M. V. Mirkin, Anal. Chem., 2018, 90, $8285-8289$

18 J. Dunevall, H. Fathali, N. Najafinobar, J. Lovric, J. Wigström, A.S. Cans and A. G. Ewing, J. Am. Chem. Soc., 2015, 137, 4344-4346.

19 Y. E. Jeun, B. Baek, M. W. Lee and H. S. Ahn, Chem. Commun., 2018, 54, 10052-10055.

20 J. H. Park and H. S. Ahn, Appl. Surf. Sci., 2020, 504, 144517.

21 M. W. Glasscott, A. D. Pendergast and J. E. Dick, ACS Appl. Nano Mater., 2018, 1, 5702-5711.

22 A. D. Pendergast, M. W. Glasscott, C. Renault and J. E. Dick, Electrochem. Commun., 2019, 98, 1-5.

23 M. W. Glasscott, A. D. Pendergast, S. Goines, A. R. Bishop, A. T. Hoang, C. Renault and J. E. Dick, Nat. Commun., 2019, 10, 2650.

24 Y. E. Jeun, J. H. Park, J. Y. Kim and H. S. Ahn, Chem. - Eur. J., 2020, 26, 4039-4043.
25 J. Xia, N. Masaki, M. Lira-Cantu, Y. Kim, K. Jiang and S. Yanagida, J. Am. Chem. Soc., 2008, 130, 1258-1263.

26 B.-w. Park, M. Pazoki, K. Aitola, S. Jeong, E. M. J. Johansson, A. Hagfeldt and G. Boschloo, ACS Appl. Mater. Interfaces, 2014, 6, 2074-2079.

27 C. Yuan, S. Guo, S. Wang, L. Liu, W. Chen and E. Wang, Ind. Eng. Chem. Res., 2013, 52, 6694-6703.

28 G.-F. Wang, X.-M. Tao and R.-X. Wang, Compos. Sci. Technol., 2008, 68, 2837-2841.

29 J. H. Kim, C. W. Joo, J. Lee, Y. K. Seo, J. W. Han, J. Y. Oh, J. S. Kim, S. Yu, J. H. Lee, J.-I. Lee, C. Yun, B. H. Choi and Y. H. Kim, Macromol. Rapid Commun., 2016, 37, 1427-1433.

30 Y. Li, D. Wang, X. Pei, Z. Shi, L. Wang, W. Zhang and J. Jin, Soft Matter, 2011, 7, 2682-2686.

31 Y. Zhang and K. S. Suslick, Chem. Mater., 2015, 27, 7559-7563.

32 Y. Liu, A. P. F. Turner, M. Zhao and W. C. Mak, Eur. Polym. J., 2018, 99, 332-339.

33 R. Asami, M. Atobe and T. Fuchigami, J. Am. Chem. Soc., 2005, 127, 13160-13161.

34 K. Nakabayashi, F. Amemiya, T. Fuchigami, K. Machida, S. Takeda, K. Tamamitsu and M. Atobe, Chem. Commun., 2011, 47, 5765-5767.

35 E. Poverenov, M. Li, A. Bitler and M. Bendikov, Chem. Mater., 2010, 22, 4019-4025.

36 T. Lindfors, Z. A. Boeva and R.-M. Latonen, $R S C A d v$., 2014, 4, 25279-25286.

37 Y. Lu, C. Kacica, S. Bansal, L. M. Santino, S. Acharya, J. Hu, C. Izima, K. Chrulski, Y. Diao, H. Wang, H. Yang, P. Biswas, J. Schaefer and J. M. D'Arcy, ACS Appl. Mater. Interfaces, 2019, 11, 47320-47329.

38 K. Muro, M. Watanabe, T. Tamai, K. Yazawa and K. Matsukawa, RSC Adv., 2016, 6, 87147-87152.

39 Y.-M. Xiao, J.-Y. Lin, J.-H. Wu, S.-Y. Tai and G.-T. Yue, Electrochim. Acta, 2012, 83, 221-226.

40 L. Groenendaal, G. Zotti, P.-H. Aubert, S. M. Waybright and J. R. Reynolds, Adv. Mater., 2003, 15, 855-879.

41 B. R. Pistillo, K. Menguelti, N. Desbenoit, D. Arl, R. Leturcq, O. M. Ishchenko, M. Kunat, P. K. Baumann and D. Lenoble, J. Mater. Chem. C, 2016, 4, 5617-5625.

$42 \mathrm{~J} . \mathrm{Li}$ and Y. Ma, Synth. Met., 2016, 217, 185-188.

43 J. Metsik, K. Saal, U. Mäeorg, R. Lõhmus, S. Leinberg, H. Mändar, M. Kodu and M. Timusk, J. Polym. Sci., Part B: Polym. Phys., 2014, 52, 561-571.

44 M. Culebras, C. M. Gómez and A. Cantarero, J. Mater. Chem. A, 2014, 2, 10109-10115.

45 W. Fan, C.-Y. Guo and G. Chen, J. Mater. Chem. A, 2018, 6, 12275-12280. 46 A. Savva, S. Wustoni and S. Inal, J. Mater. Chem. C, 2018, 6, 12023-12030.

47 S. Fabiano, N. Sani, J. Kawahara, L. Kergoat, J. Nissa, I. Engquist, X. Crispin and M. Berggren, Sci. Adv., 2017, 3, e1700345.

48 K. Tybrandt, I. V. Zozoulenko and M. Berggren, Sci. Adv., 2017, 3, eaao3659.

49 H. Zhou, G. Han, Y. Chang, D. Fu and Y. Xiao, J. Power Sources, 2015, 274, 229-236.

50 H. Zhou, X. Zhi and H.-J. Zhai, Org. Electron., 2018, 63, 149-158.

51 D. Ni, Y. Chen, H. Song, C. Liu, X. Yang and K. Cai, J. Mater. Chem. A, 2019, 7, 1323-1333.

52 X. Zhang and P. Samorì, ChemNanoMat, 2017, 3, 362-372.

53 T. Earmme and S. A. Jenekhe, J. Mater. Chem., 2012, 22, 4660-4668.

54 L. Qian, Y. Zheng, J. Xue and P. H. Holloway, Nat. Photonics, 2011, 5, 543-548.

55 A. N. Aleshin, S. R. Williams and A. J. Heeger, Synth. Met., 1998, 94, 173-177.

56 A. N. Aleshin, R. Kiebooms and A. J. Heeger, Synth. Met., 1999, 101, 369-370.

57 M. N. Gueye, A. Carella, J. Faure-Vincent, R. Demadrille and J.-P. Simonato, Prog. Mater. Sci., 2020, 108, 100616. 